\title{
Cloroquina e hidroxicloroquina: uso off-label em processos judiciais no estado de Minas Gerais
}

Chloroquine and hydroxychloroquine: off-label use in lawsuits in the State of Minas Gerais, Brazil

Cloroquina e hidroxicloroquina: uso fuera de lo indicado en demandas en el estado de Minas Gerais, Brasil

Patrícia de Oliveira ${ }^{1}$

Camila Cátia Vilela Viana ${ }^{2}$

Orozimbo Henriques Campos Neto $^{3}$

Giovana Gonçalves Pereira ${ }^{4}$

André Soares Santos 5

Keli Bahia Felicíssimo Zocratto ${ }^{6}$

\section{Resumo}

Objetivo: analisar os processos judiciais relacionados à cloroquina e hidroxicloroquina, segundo indicações e registros na Agência Nacional de Vigilância Sanitária (Anvisa), bem como nos Protocolos Clínicos e Diretrizes Terapêuticas (PCDT), considerando as doenças que motivaram os pleitos, sob o olhar da bioética para o uso off-label. Metodologia: estudo documental, exploratório-descritivo, transversal, quantitativo. Foram analisados os processos judiciais por hidroxicloroquina e cloroquina, nos últimos 20 anos em Minas Gerais. Realizou-se a análise descritiva, considerando as seguintes variáveis: doença; CID-10; medicamentos; ano de incorporação no componente especializado da assistência farmacêutica (CEAF); e ano da ação judicial. Foi utilizado o software SPSS® versão 19 na análise dos dados. Resultados: dos 155 processos analisados, 148 (95,5\%) apresentaram demanda por hidroxicloroquina 400mg e 7 (4,5\%) por cloroquina $250 \mathrm{mg}$. Os medicamentos analisados possuíam registros na Anvisa e o uso off-label foi constatado em $8,1 \%$ para

\footnotetext{
${ }^{1}$ Mestranda em Gestão de Serviço de Saúde, Universidade Federal de Minas Gerais, Belo Horizonte, MG, Brasil; coordenadora, Assistência Farmacêutica, Superintendência Regional de Saúde de Belo Horizonte, Secretaria de Estado de Saúde de Minas Gerais, Belo Horizonte, MG, Brasil. https://orcid.org/0000-0002-6116-7889. E-mail: patricia.oliveirapop@gmail.com

${ }_{2}^{2}$ Mestranda em Gestão de Serviço de Saúde, Universidade Federal de Minas Gerais, Belo Horizonte, MG, Brasil; diretora de Regulação de Urgência e Emergência, Secretaria de Estado de Saúde de Minas Gerais, Belo Horizonte, MG, Brasil. https://orcid.org/0000-0001-7659-9310.E-mail: camilamtzenfe@yahoo.com.br

${ }^{3}$ Doutor em Saúde Pública, Universidade Federal de Minas Gerais, Belo Horizonte, MG, Brasil; professor, Faculdade Ciências da Vida/Instituto Vida e Saúde, Sete Lagoas, Minas Gerais, Brasil. https://orcid.org/0000-0001-6415-3709. E-mail: orozimbo1984@gmail.com

${ }^{4}$ Mestranda em Gestão de Serviço de Saúde, Universidade Federal de Minas Gerais, Belo Horizonte, MG, Brasil; coordenadora, Assistência Farmacêutica, Superintendência Regional de Saúde de Pirapora, Secretaria de Estado de Saúde de Minas Gerais, Pirapora, MG, Brasil. https://orcid.org/0000-0002-0479-793X. E-mail: giovanagpereira@yahoo.com.br

${ }^{5}$ Doutor em Medicamentos e Assistência Farmacêutica; residente pós-doutoral, Universidade Federal de Minas Gerais, Belo Horizonte, MG, Brasil. https://orcid.org/000-0002-2856-7100. E-mail: andresantos111@ufmg.br

6 Doutora em Saúde Pública, Universidade Federal de Minas Gerais, Belo Horizonte, MG, Brasil; professora adjunta, Departamento de Gestão em Saúde (DGES), Universidade Federal de Minas Gerais. https://orcid.org/0000-0002-57420570. E-mail: kelibahia@yahoo.com.br
} 
hidroxicloroquina e 14,3\% para cloroquina. Discussão: as demandas judiciais para a hidroxicloroquina e cloroquina estiveram relacionadas às suas indicações registradas em bula e dentro dos PCDT. No entanto, a presença no estudo de seu uso off-label demonstrou que o Judiciário defere os pleitos, trazendo implicações bioéticas ao impactar nos princípios da autonomia, não maleficência, beneficência e justiça. Conclusão: a judicialização de medicamento off-label interfere no planejamento, execução e financiamento das políticas públicas de saúde, além de causar exposição do cidadão a riscos desconhecidos, ao utilizar medicamentos com indicações não amparadas pelas evidências científicas, pela Anvisa e pela própria indústria produtora do medicamento.

\title{
Palavras-chave
}

Judicialização da saúde. Bioética. Hidroxicloroquina. Cloroquina.

\begin{abstract}
Objective: to analyze the lawsuits related to chloroquine and hydroxychloroquine, according to indications and records at the National Health Surveillance Agency (Anvisa) as well as in the Clinical Protocols and Therapeutic Guidelines (PCDT) considering the diseases that motivated the claims, under the view of the bioethics for off-label use. Methodology: documentary, exploratory-descriptive, transversal, quantitative study. The hydroxychloroquine and chloroquine lawsuits in the last 20 years in Minas Gerais were analyzed. Descriptive analysis was performed by considering the following variables: disease; ICD-10; medicines; year of incorporation into the specialized pharmaceutical assistance component (CEAF); and year of the lawsuit. SPSS® software version 19 was used to analyze the data. Results: the corpus of 155 processes analyzed, 148 (95.5\%) presented demand for $400 \mathrm{mg}$ hydroxychloroquine and 7 (4.5\%) for $250 \mathrm{mg}$ chloroquine. The analyzed drugs were registered with Anvisa and off-label use was found in $8.1 \%$ for hydroxychloroquine and $14.3 \%$ for chloroquine. Discussion: the judicial demands for hydroxychloroquine and chloroquine were related to their indications registered in the package leaflet and within the PCDT. However, the presence in the study of its off-label use demonstrated that the Judiciary grants the claims, bringing bioethical implications by impacting the principles of autonomy, non-maleficence, beneficence, and justice. Conclusion: the judicialization of off-label medicine interferes in the planning, execution and financing of public health policies, in addition to causing citizen exposure to unknown risks, when using medicines with indications not supported by scientific evidence, by Anvisa and by the production industry itself of the medicine.
\end{abstract}

\section{Keywords}

Health's judicialization. Bioethics. Hydroxychloroquine. Chloroquine.

\section{Resumen}

Objetivo: analizar los juicios relacionados con la cloroquina y la hidroxicloroquina, de acuerdo con las indicaciones y registros de la Agencia Nacional de Vigilancia Sanitaria (Anvisa), así como en los Protocolos Clínicos y Guías Terapéuticas (PCDT), considerando las enfermedades que motivaron las reclamaciones, bajo la óptica de la bioética para uso no indicado en la etiqueta. Metodología: estudio documental, exploratorio-descriptivo, transversal, cuantitativo. Se analizaron los juicios de hidroxicloroquina y cloroquina de los últimos 20 años en Minas Gerais. Se realizó un análisis descriptivo, considerando las siguientes variables: enfermedad; ICD-10; medicamentos; año de incorporación al componente de asistencia farmacéutica especializada (CEAF); y año de la demanda. Se utilizó el software SPSS $\AA$ versión 19 para analizar los datos. Resultados: de los 155 procesos analizados, 148 (95,5\%) presentaron demanda de $400 \mathrm{mg}$ de hidroxicloroquina y 
$7(4,5 \%)$ de $250 \mathrm{mg}$ de cloroquina. Los medicamentos analizados se registraron en Anvisa y el uso fuera de lo indicado se encontró en $8.1 \%$ para hidroxicloroquina y $14.3 \%$ para cloroquina. Discusión: las demandas judiciales de hidroxicloroquina y cloroquina estaban relacionadas con sus indicaciones registradas en el prospecto y dentro del PCDT. Sin embargo, la presencia en el estudio de su uso fuero de lo indicado demostró que el Poder Judicial otorga los reclamos, trayendo implicaciones bioéticas al incidir en los principios de autonomía, no maleficencia, beneficencia y justicia. Conclusión: la judicialización de los medicamentos fuera de lo indicdo interfiere en la planificación, ejecución y financiamiento de las políticas de salud pública, además de provocar la exposición ciudadana a riesgos desconocidos, al utilizar medicamentos con indicaciones no sustentadas en evidencia científica, por Anvisa y por la propia industria productora. de la medicina.

\section{Palabras clave}

Judicialización de la salud. Bioética. Hidroxicloroquina. Cloroquina

\section{Introdução}

A cloroquina e a hidroxicloroquina são aminoquinolinas de ação antimalárica, registradas no Brasil para tratamento de doenças autoimunes como lúpus e artrite reumatoide (1). Novos ensaios clínicos são necessários para se realizar o reposicionamento ou reaproveitamento desses medicamentos para novas indicações terapêuticas que ainda não estejam aprovadas. A avaliação clínica de reposicionamento pode representar uma boa estratégia quando o meio científico enfrenta o desafio de encontrar medicamentos seguros e eficazes para infecções virais emergentes que se expandem, causando um número crescente de óbitos $(2,3)$.

Para a utilização de medicamentos de forma segura as evidências disponíveis sobre seus benefícios na utilização devem ser suficientes e, enquanto não haja evidências científicas de melhor qualidade sobre a eficácia e segurança dos medicamentos avaliados, é recomendável que eles sejam usados apenas no contexto de estudos devidamente registrados, aprovados e eticamente aceitáveis (4). Em 2003, foi lançada a hipótese de que a cloroquina poderia ser útil para tratar a síndrome respiratória aguda grave (SARS), um ano após o vírus SARS-CoV ter sido identificado como o agente etiológico da SARS (2).

Em 2020, um universo integralmente novo - relacionado à pandemia da Covid-19, doença causada pelo coronavírus SARS-CoV-2 - se caracterizou como uma emergência em saúde pública de importância internacional, conforme apontada pela Organização Mundial da Saúde (OMS) (5). No contexto da saúde pública brasileira, o Sistema Único de Saúde (SUS) se depara com mais uma situação adversa para cumprir os princípios doutrinários do direito constitucional à saúde: universalidade, equidade e integralidade. A complexidade do momento impõe desafios adicionais à vigilância epidemiológica, às relações internacionais 
e à programação de políticas públicas, sobretudo por meio de medidas que reduzam as desigualdades de acesso às ações e serviços de saúde (6).

Desde 16 de março de 2020, quando pesquisadores franceses publicaram que 0 medicamento hidroxicloroquina curou pacientes com a Covid-19, os quais se encontravam em leitos de UTI, a esperança pela cura superou qualquer racionalidade sobre os possíveis efeitos adversos (2). Dilemas como esses alcançam os operadores do direito no Ministério Público e no Judiciário, que precisam se posicionar para determinar ações que, em teoria, correspondam ao cumprimento do direito à saúde pelo SUS.

Nos últimos anos, tem-se observado uma explosão de processos judiciais por prestações individuais, solicitações de medicamentos, procedimentos e equipamentos para a saúde. As decisões judiciais atreladas a esses processos podem resolver uma situação pontual e individual, mas não modificam o sistema em termos de aprimoramento ou correção das políticas públicas. A realocação de recursos públicos para o cumprimento de decisões judiciais acirra a disputa com as políticas de saúde, uma vez que pode favorecer o autor do processo judicial, penalizando a coletividade que utiliza o SUS (7). A pandemia da Covid-19 traz à tona o conhecido desafio enfrentado pelo Judiciário que, ao evidenciar as decisões vinculadas a processos de caráter individual e para a utilização de medicamentos sem comprovação científica, podem trazer distorções frente à equidade, especialmente quando se está diante de uma doença que afeta toda a população em termos sociais, econômicos e sanitários, podendo inclusive trazer agravos à saúde da população brasileira $(8,9)$.

O presente estudo buscou trabalhar o caso concreto dos processos judiciais pelos medicamentos cloroquina e hidroxicloroquina, no estado de Minas Gerais, de maio de 2000 a maio de 2020, com abrangência em período anterior e no momento da pandemia da Covid19. O objetivo foi analisar os processos judiciais relacionados às referidas terapias farmacológicas, conforme os registros desses medicamentos na Agência Nacional de Vigilância Sanitária (Anvisa), as categorias de registros realizados pelos laboratórios (genérico, similar e referência), bem como a padronização nos Protocolos Clínicos e Diretrizes Terapêuticas (PCDT), considerando as doenças que motivaram os pleitos judiciais. Nessa trajetória, um olhar sobre o uso off-label norteou o estudo para que o debate acerca dos fundamentos da bioética aplicada compusesse o artigo. 


\section{Metodologia}

Estudo documental, exploratório-descritivo, transversal, de caráter quantitativo, cujo objeto de análise corresponde às demandas por hidroxicloroquina e cloroquina requisitadas por meio de processos judiciais, em que o estado de Minas Gerais e seus representantes figuram como réus.

Os dados foram obtidos mediante análise da relação dos medicamentos judicializados dos últimos 20 anos (31 de maio de 2000 a 31 de maio de 2020), pela Secretaria de Estado de Saúde de Minas Gerais (SES/MG). A relação dos medicamentos foi disponibilizada pela Coordenação de Planejamento e Distribuição do Núcleo de Judicialização em Saúde (CPD/NJS) da SES/MG, em uma planilha do Microsoft Office Excel 2016, contendo o registro dos processos judiciais. Foram selecionados os processos que continham os princípios ativos difosfato de cloroquina e sulfato de hidroxicloroquina. Realizou-se a identificação das dosagens, forma farmacêutica e categoria do registro pelo laboratório produtor (genérico, similar e referência), para cada princípio ativo.

Com base nos processos judiciais selecionados, foram realizadas consultas no Sistema de Gerenciamento da Assistência Farmacêutica da Judicialização (SIGAFJUD) sistema de registro das informações relacionadas às demandas judiciais em saúde do estado de Minas Gerais - para busca das informações sobre a doença e sua respectiva Classificação Internacional de Doenças (CID.10).

Para a identificação do uso off-label da cloroquina e hidroxicloroquina, utilizou-se a bula profissional e o registro dos seus princípios ativos. Verificou-se a existência de registros vigentes de cada um dos princípios ativos na Anvisa, por meio de pesquisa no banco de dados Consulta a Medicamentos e Hemoderivados, disponível no sítio eletrônico https://consultas.anvisa.gov.br/\#/medicamentos/. A pesquisa foi realizada no dia 20 de junho de 2020. Para verificar as indicações terapêuticas registradas, foi acessada a bula profissional para cada medicamento no Bulário eletrônico da Anvisa (http://www.anvisa.gov.br/datavisa/fila_bula/index.asp).

A análise descritiva, por meio de frequências absolutas e relativas, traçou o perfil dos processos judiciais por difosfato de cloroquina e sulfato de hidroxicloroquina, utilizando as seguintes variáveis: a doença; código na CID-10; medicamentos (dosagem, forma farmacêutica e categoria do registro pelo laboratório produtor); ano de incorporação no PCDT do medicamento por código da CID-10; e ano da ação judicial. Foi utilizado o software SPSS® versão 19 na análise dos dados e a apresentação foi feita em tabela e gráfico. 


\section{Resultados}

No período de 31 de maio 2000 a 31 de maio de 2020, foram encontrados 155 processos judiciais para os medicamentos estudados, dentre os quais, $148(95,5 \%)$ com demanda por sulfato de hidroxicloroquina $400 \mathrm{mg}$, comprimido revestido; e sete $(4,5 \%)$ por difosfato de cloroquina 250mg, comprimido (Tabela 1).

Tabela 1. Perfil dos pleitos judiciais por cloroquina e hidroxicloroquina (maio/2000 a maio/2020)

\begin{tabular}{l|c|c}
\hline \multicolumn{1}{c|}{ Medicamento } & $\mathbf{n}$ & $\%$ \\
\hline Sulfato de hidroxicloroquina 400mg & 148 & 95,5 \\
\hline Difosfato de cloroquina 250mg & 7 & 4,5 \\
\hline Total & $\mathbf{1 5 5}$ & $\mathbf{1 0 0}$ \\
\hline
\end{tabular}

Fonte: banco de dados dos medicamentos judicializados contra a SES-MG (2000-2020).

A primeira demanda judicial para os medicamentos estudados foi registrada em outubro de 2005, seguindo uma distribuição disforme ao longo do período analisado. Entre os anos de 2013 a 2019, foram ajuizadas 126 (85,14\%) processos judiciais por esses medicamentos, tendo o maior registro de volume anual no ano de $2014(n=34)$, relacionado ao pleito exclusivo de hidroxicloroquina. Não houve registro de processos judiciais por esses medicamentos no ano de 2020 (Gráfico 1).

Gráfico 1. Evolução das ações judiciais por difosfato de cloroquina e sulfato de hidroxicloroquina no estado de Minas Gerais (maio/2000 a maio/2020)

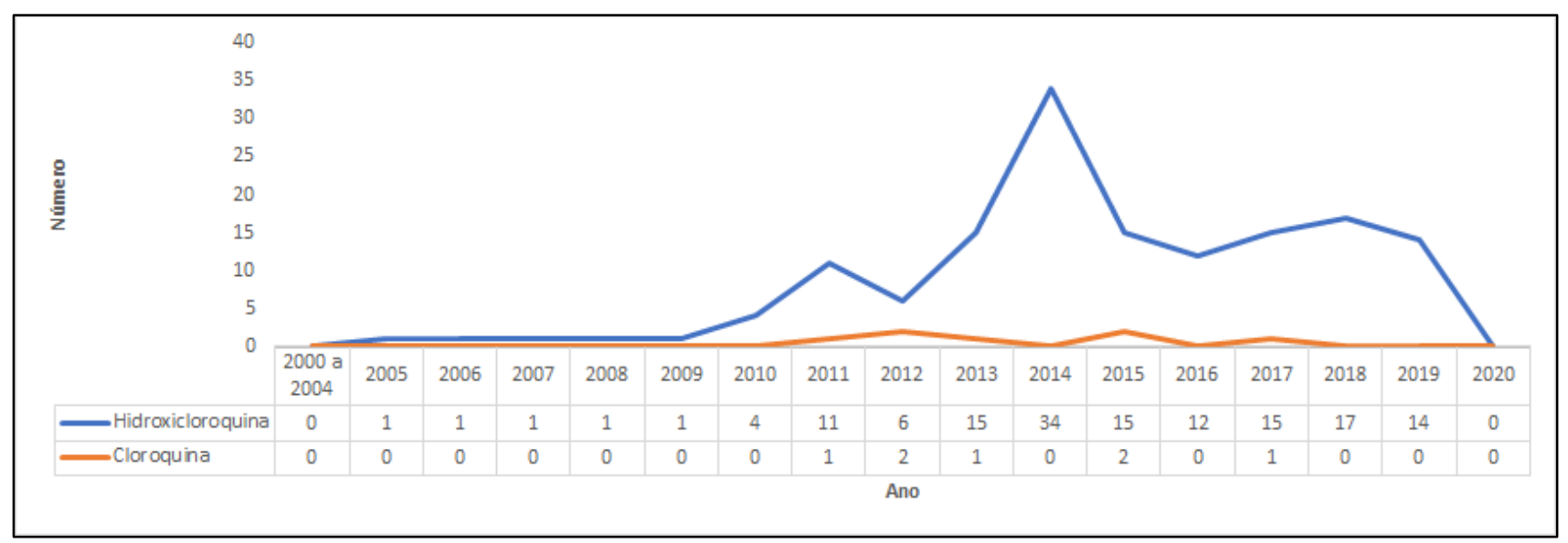

Fonte: Sistema de Gerenciamento da Assistência Farmacêutica da Judicialização (SIGAFJUD), SES/MG. 
Considerando $\mathrm{o}$ ano de registro do processo judicial e a disponibilidade dos medicamentos analisados no SUS no mesmo ano, observou-se que, em três demandas $(1,9 \%)$, o medicamento pleiteado não estava disponível no SUS, no componente especializado da assistência farmacêutica (CEAF). Essas três ações foram ajuizadas em 2005, 2006 e 2007. Em 29,7\% ( $n=46)$ dos processos, entre os anos de 2008 a 2018, os medicamentos estavam padronizados no SUS, mas a doença do demandante não estava contemplada nos PCDT, publicados pelo Ministério da Saúde. Havia a disponibilidade no SUS, de acordo com os PCDT, para 81 (52,3\%) dos processos judiciais pelos medicamentos analisados. Por fim, em $25(16,1 \%)$ demandas analisadas, não foi possível cruzar a informação sobre a disponibilidade do item no SUS e o CID-10, por não estar identificada a doença do requerente (Tabela 2).

Tabela 2. Perfil dos pleitos judiciais por cloroquina e hidroxicloroquina e por período de disponibilização no CEAF do SUS, conforme vigência do PCDT ou Nota Técnica (maio/2000 a maio/2020).

\begin{tabular}{|c|c|c|c|c|c|c|c|c|c|c|}
\hline \multirow{3}{*}{$\begin{array}{c}\text { Disponibilidade } \\
\text { no SUS no } \\
\text { CEAF e PCDT }\end{array}$} & \multicolumn{2}{|c|}{$2000^{*}$ a 2007} & \multicolumn{2}{|c|}{2008 a 2012} & \multicolumn{2}{|c|}{2013 a 2019} & \multicolumn{2}{|c|}{$2020^{*}$} & \multirow{2}{*}{\multicolumn{2}{|c|}{ TOTAL }} \\
\hline & \multicolumn{2}{|c|}{ Sem PCDT } & \multicolumn{2}{|c|}{$\begin{array}{l}\text { PCDT: } \\
\text { Artrite }\end{array}$} & \multicolumn{2}{|c|}{$\begin{array}{c}\text { PCDT: } \\
\text { Artrite/Lupus }\end{array}$} & \multicolumn{2}{|c|}{$\begin{array}{c}\text { PCDT: } \\
\text { Artrite/Lupus } \\
\text { Nota Técnica: } \\
\text { Covid-19 }\end{array}$} & & \\
\hline & $\mathbf{n}$ & $\%$ & $\mathbf{n}$ & $\%$ & $\mathbf{n}$ & $\%$ & $\mathbf{n}$ & $\%$ & $\mathbf{n}$ & $\%$ \\
\hline $\begin{array}{l}\text { Indisponível no } \\
\text { SUS }\end{array}$ & 3 & 100 & 0 & 0 & 0 & 0 & 0 & 0 & 3 & 1,9 \\
\hline $\begin{array}{l}\text { Indisponível no } \\
\text { PCDT }\end{array}$ & 0 & 0 & 20 & 76,9 & 26 & 20,6 & 0 & 0 & 46 & 29,7 \\
\hline $\begin{array}{ll}\text { Disponível no } \\
\text { PCDT }\end{array}$ & 0 & 0 & 2 & 7,7 & 79 & 62,7 & 0 & 0 & 81 & 52,3 \\
\hline Sem informação & 0 & 0 & 4 & 15,4 & 21 & 16,7 & 0 & 0 & 25 & 16,1 \\
\hline Total & 3 & 100 & 26 & 100 & 126 & 100 & 0 & 0 & 155 & 100 \\
\hline
\end{tabular}

Fonte: banco de dados dos medicamentos judicializados contra a SES-MG (2000-2020).

*maio de 2000 e maio 2020

Os medicamentos analisados neste estudo possuíam, até 20 de junho de 2020, registros ativos na Anvisa. Foram encontrados seis registros válidos de cinco diferentes laboratórios privados para o sulfato de hidroxicloroquina 400mg (Sanofi, Apsen Farmacêutica, Germed Farmacêutica, Eurofarma Laboratórios e EMS S/A) e três registros válidos de três diferentes laboratórios para o difosfato de cloroquina $250 \mathrm{mg}$, sendo dois 
laboratórios públicos (Fundação Oswaldo Cruz e Laboratório Químico Farmacêutico do Exército) e um privado (Cristália Produtos Químicos Farmacêuticos Ltda.).

De acordo com as bulas para os profissionais de saúde, as indicações aprovadas e registradas pela Anvisa para ambos medicamentos foram: afecções reumáticas e dermatológicas (reumatismo e problemas de pele); artrite reumatoide (inflamação crônica das articulações); artrite reumatoide juvenil (em crianças); lúpus eritematoso sistêmico (doença multissistêmica); lúpus eritematoso discoide (lúpus eritematoso da pele); condições dermatológicas (problemas de pele) provocadas ou agravadas pela luz solar; malária (doença causada por protozoários), para tratamento das crises agudas e tratamento supressivo de malária por Plasmodium vivax, P. ovale, P. malariae e cepas (linhagens) sensíveis de $P$. falciparum (protozoários causadores de malária) e tratamento radical da malária provocada por cepas sensíveis de $P$. falciparum.

Entre os 155 processos judiciais analisados, 148 tratavam de pleito por hidroxicloroquina, sendo que $112(75,7 \%)$ apresentaram demandas relacionadas a tratamento de doenças com indicações registrados em bula e $12(8,1 \%)$ tratavam de uso offlabel. Em 24 (16,2\%) processos judiciais, não foi possível identificar nos registros analisados a doença do demandante. Em relação à cloroquina, dos 7 processos judiciais pesquisados, $57,1 \%(n=4)$ eram demandas relacionadas a tratamento de doenças com indicações registrados em bula; $14,3 \%(n=1)$ tratavam de uso off-label; e em $28,6 \%(n=2 \%)$ dos processos judiciais não foi possível identificar a doença do demandante nos registros analisados (Tabela 3).

Tabela 3. Perfil dos pleitos judiciais por cloroquina e hidroxicloroquina, conforme registro da indicação dos medicamentos (maio/2000 a maio/2020)

\begin{tabular}{l|c|c|c|c}
\hline \multirow{2}{*}{ Indicação em bula pela Anvisa } & \multicolumn{2}{|c|}{ Hidroxicloroquina } & \multicolumn{2}{c}{ Cloroquina } \\
& $\mathbf{n}$ & $\%$ & $\mathbf{n}$ & $\%$ \\
\hline Sem indicação (Off label) & 12 & 8,1 & 1 & 14,3 \\
\hline Sem informação suficiente & 24 & 16,2 & 2 & 28,6 \\
\hline Com indicação & 112 & 75,7 & 4 & 57,1 \\
\hline Total & $\mathbf{1 4 8}$ & $\mathbf{1 0 0}$ & $\mathbf{7}$ & 100 \\
\hline
\end{tabular}

Fonte: banco de dados dos medicamentos judicializados contra a SES-MG (2000-2020). 
Nas demandas por sulfato de hidroxicloroquina $400 \mathrm{mg}$, em relação à exigência por laboratório fabricante, $101(68,2 \%)$ processos judiciais não definiram laboratório para o cumprimento da decisão; 42 (28,4\%) exigiam que a demanda fosse cumprida com o medicamento registrado na categoria de similar do laboratório Apsen; e $5(3,4 \%)$ com o medicamento referência produzido pelo laboratório Sanofi. Para as demandas por difosfato de cloroquina 250mg não houve especificação de marcas (Tabela 4).

Tabela 4. Perfil dos pleitos judiciais por cloroquina e hidroxicloroquina, conforme categoria de registro na Anvisa (maio/2000 a maio/2020)

\begin{tabular}{l|c|c|c|c}
\hline \multirow{2}{*}{ Categoria do Registro pela Anvisa } & \multicolumn{2}{|c|}{ Hidroxicloroquina } & \multicolumn{2}{c}{ Cloroquina } \\
\cline { 2 - 5 } & $\mathbf{n}$ & $\%$ & $\mathbf{n}$ & $\%$ \\
\hline Medicamento Referência & 5 & 3,4 & 0 & 0 \\
\hline Medicamento Similar & 42 & 28,4 & 0 & 0 \\
\hline Sem Definição & 101 & 68,2 & 7 & 100 \\
\hline Total & $\mathbf{1 4 8}$ & $\mathbf{1 0 0}$ & $\mathbf{7}$ & $\mathbf{1 0 0}$ \\
\hline
\end{tabular}

Fonte: Banco de Dados dos Medicamentos Judicializados contra a SES-MG (2000-2020)

\section{Discussão}

A presença de processos judiciais pleiteando a hidroxicloroquina foi anterior ao da cloroquina, sendo que a maioria dos medicamentos pleiteados estavam inseridos no PCDT e disponibilizados no CEAF. A definição de marca e laboratório, assim como o uso off-label foram observadas nas decisões analisadas, exceto no período da pandemia. As decisões judiciais acabam por fixar políticas públicas na saúde, fazendo que o Judiciário, ao atuar como promotor da garantia de direitos passe a assumir o papel de administrador do sistema com uma visão restrita, impactando no atendimento aos princípios da bioética.

A maioria das demandas judiciais foi para o medicamento hidroxicloroquina $400 \mathrm{mg}$, sendo que os processos judiciais para ambos os medicamentos foram entre os anos de 2005 a 2019. Não houve demanda judicial no ano de 2020 para nenhum dos medicamentos estudados, mesmo com toda repercussão na atribuição aos referidos medicamentos potenciais propriedades no combate à infecção humana pelo novo coronavírus, inclusive por orientação do Ministério da Saúde (10). Tal situação gerou um desabastecimento dos 
medicamentos nas farmácias e drogarias, dificultando o acesso aos pacientes com acometimentos de saúde que possuem indicação médica para utilizá-los (11).

Em relação à contemplação e disponibilização no SUS por meio dos PCDT do CEAF, os medicamentos pleiteados nos processos em sua grande maioria estavam inseridos no PCDT e disponibilizadas pela Assistência Farmacêutica. A cloroquina e a hidroxicloroquina, que atualmente fazem parte de estudos clínicos para avaliação da indicação no tratamento da Covid-19, estão incorporadas e disponibilizadas no SUS por meio do CEAF desde 2008 para o tratamento de artrite reumatoide. A partir de 2013, houve a incorporação e disponibilização da hidroxicloroquina e cloroquina no PCDT de lúpus eritematoso sistêmico (12).

Atualmente, a cloroquina também é incorporada no SUS como opção terapêutica para o tratamento de pacientes com malária, doença de perfil endêmico no Brasil e que demanda a inclusão de tratamento farmacológico no CEAF (13). Em 2020, o Ministério da Saúde disponibiliza, sem submissão à Comissão Nacional de Incorporação de Tecnologias (Conitec) no SUS, a cloroquina como terapia adjuvante no tratamento de formas graves da Covid-19 por meio da Nota Informativa ํㅡ 5/2020-DAF/SCTIE/MS e, posteriormente, orienta o manuseio medicamentoso precoce de pacientes com diagnóstico da Covid-19, por meio da publicação da Nota Informativa no 9/2020-SE/GAB/SE/MS (14).

No Brasil, desde 2011, foi atribuída à Conitec a responsabilidade de incorporação, exclusão ou alteração de medicamentos, produtos e procedimentos ofertados pelo SUS, bem como a constituição ou a alteração dos PCDT (15). Para a avaliação dessas tecnologias, são consideradas a existência de evidências científicas de eficácia, efetividade e segurança da tecnologia avaliada em comparação às demais, bem como o impacto orçamentário da incorporação (16). A Avaliação de Tecnologias em Saúde (ATS) assumiu um papel de destaque no sistema de saúde nos últimos anos, principalmente frente à limitação de recursos que se agravou após a Emenda Constitucional n 95/2016, responsável por alterações que implicam na redução do orçamento destinado à saúde pública $(17,18)$.

A presença de indicação de marca do medicamento para cumprimento do processo judicial, que define assim o laboratório produtor do item, foi observada em alguns processos analisados no presente estudo. Diante do cenário de restrição de recursos financeiros imposto pela EC no 95/2016, a definição de marca impõe uma seleção prévia e direciona o processo licitatório, com vedação prevista na Lei no 8.666/1993, e fere os princípios legais 
da isonomia, da impessoalidade, da moralidade e da probidade administrativa. A legislação que institui o medicamento genérico no país - a Lei Federal no 9.787/19 - estabelece que a prescrição de medicamentos pela rede pública deve ser realizada utilizando a Denominação Comum Brasileira (DCB), ou seja, a utilização da denominação do fármaco ou princípio farmacologicamente ativo. A prescrição e a consequente solicitação judicial por uma marca específica deveriam ser feitas somente em casos excepcionais, mediante notificação junto à Anvisa da ocorrência de efeitos adversos ou inefetividade terapêutica, conforme Resolução Anvisa $n^{\circ}$ 4, de 10 de fevereiro de 2009 (19).

O presente estudo evidenciou que, dentre os processos judiciais analisados existem demandas com uso sem indicação registradas em bula pela Anvisa, denominado uso offlabel. Tal fato demonstra que o Judiciário nem sempre defere os pleitos judiciais amparado pelas evidências científicas, pelas normativas da Anvisa, e pela própria indústria produtora do medicamento, criando uma jurisprudência perigosa, ao considerar somente a prescrição médica e desconsiderar possíveis falhas ou influências na mesma (7). A não observância, pelo Judiciário, da evidência científica ao deferir pedidos de solicitação de medicamentos uso off-label ou não inseridos nos PCDT desperta questionamentos sobre o prisma dos princípios da bioética, como a autonomia, não maleficência, beneficência e justiça.

A autonomia refere à autodeterminação do indivíduo de tomar decisões que afetam sua vida, sua integridade física e psíquica e suas relações sociais. É a capacidade de decidir e agir livre e independentemente, após receber informações claras sobre os benefícios, contra-indicações, potenciais efeitos colaterais para sua decisão, muitas vezes documentado no termo de consentimento informado livre e esclarecido (TCILE). O TCILE também chamado de termo de esclarecimento e responsabilidade (TER) - reflete, em particular, o direito moral da pessoa à integridade corporal e o direito de autonomia na participação ativa da tomada de decisões conducentes à manutenção da sua saúde e à própria adesão à terapêutica, o que pressupõe adequada informação e uma decisão livre e esclarecida (20).

A autonomia e vulnerabilidade da pessoa humana mantém-se inalienáveis, mesmo que a terapêutica seja disponibilizada pelo sistema público de saúde. Assim, o TER pode adquirir caráter de documento assertivo ao contemplar medidas protetivas ao paciente (21). No SUS, quando o medicamento é contemplado no PCDT do CEAF, sua utilização é mediada pela aplicação obrigatória do TER, de modo a garantir a participação consciente do 
paciente na tomada de decisão sobre o seu tratamento. No cenário de indicação off-label, não se pode afirmar que isso aconteça.

A não maleficência é entendida como obrigação da ação do profissional de saúde causar o menor prejuízo ou agravos à saúde do paciente, sendo consagrado por meio do aforismo hipocrático primum non nocere (primeiro não prejudicar). Já na beneficência, o profissional de saúde deve ter a convicção e informações técnicas possíveis que assegurem o tratamento ser benéfico ao paciente, comprometendo a perseguir o máximo de benefícios, reduzindo ao mínimo os possíveis danos e riscos (22). A prescrição de off-labe/muitas vezes ocorre sem a existência de ensaios clínicos, que são instrumentos capazes de avaliar a toxicidade do produto e estabelecer as dosagens adequadas para cada tratamento (23). Seus resultados visam garantir informações necessárias para a tomada de decisão do profissional de saúde de forma segura, com vistas ao atendimento dos preceitos da não maleficência e beneficência.

O princípio de justiça pressupõe a equidade, a universalidade e a distribuição justa. A judicialização de medicamentos off-label desconsidera esse princípio, ao impor ao gestor público o fornecimento de medicamento não padronizado na política pública existente. A judicialização da saúde interfere no arranjo organizacional estabelecido pela política pública e na decisão da alocação de recursos públicos no SUS, visto que o cumprimento das determinações judiciais por produtos e serviços acarreta o deslocamento orçamentário para as referidas ações (24).

\section{Considerações finais}

A judicialização da saúde de medicamentos off-label, apesar de ser uma representação pequena no estudo relacionado à cloroquina e hidroxicloroquina, demonstrou o comportamento do Judiciário como ordenador do Sistema Único de Saúde (SUS) e como co-prescritor e gera duas situações adversas. A primeira é a interferência no planejamento, execução e financiamento das políticas públicas de saúde, ao levar à realocação de recursos orçamentários que seriam aplicados em busca do atendimento universal e equânime da população. A segunda é a exposição do cidadão a riscos desconhecidos, ao deferir medicamentos com indicações não amparadas em medicina baseada em evidência, ultrapassando os princípios da bioética, como autonomia, não maleficência, beneficência e justiça. 


\section{Referências}

1. World Health Organization (WHO). ATC/DDD Index [Internet]. 2019 [acesso em 10.ago.2020]. Disponível em:

https://www.whocc.no/atc_ddd_index/?code=P01BA\&showdescription=no

2. Paumgartten FJR, Delgado IF, Pitta LR, Oliveira ACAX. Chloroquine and hydroxychloroquine repositioning in times of Covid-19 pandemics, all that glitters is not gold. Cad. Saúde Pública [Internet]. 2020 [acesso em 13.jul.2020]; 36(5):1-3. Disponível em: https://www.scielo.br/scielo.php?script=sci_arttext\&pid=S0102-

311 X2020000500503\&tlng=en.

3. Rosa SGV, Santos WC. Clinical trials on drug repositioning for Covid-19 treatment. Revista Panam Salud Publica [Internet]. 2020 [acesso em 10.ago.2020]. Disponível em: https://iris.paho.org/bitstream/handle/10665.2/51949/v44e402020.pdf?sequence=1\&isAllow ed $=y$.

4. Organização Pan-Americana da Saúde (OPAS), Organização Mundial da Saúde (OMS). Folha informativa - Covid-19 [Internet]. 2020 [acesso em 16.ago.2020]. Disponível em: https://www.paho.org/bra/index.php?option=com_content\&view=article\&id=6101:covid19\&lt emid=875\#cloroquina-hidroxicloroquina.

5. Organização Pan-Americana da Saúde (OPAS), Organização Mundial da Saúde (OMS). OMS afirma que Covid-19 é agora caracterizada como pandemia [Internet]. Brasília, 2020 [acesso em 28.jun.2020]. Disponível em: https://www.paho.org/bra/index.php?option=com_content\&view=article\&id=6120:omsafirma-que-covid-19-e-agora-caracterizada-como-pandemia\&Itemid=812

6. Rafael RMR, Neto M, Carvalho MMB, David HMSL, Acioli S, Faria MGA. Epidemiologia, políticas públicas e pandemia de Covid-19: o que esperar no Brasil? Revista Enfermagem UERJ [Internet]. 2020 [acesso em 29.jun.2020]; 28:1-6. Disponível em: https://www.epublicacoes.uerj.br/index.php/enfermagemuerj/article/view/49570/33134

7. Paixão ALS. Reflexões sobre a judicialização do direito à saúde e suas implicações no SUS. Ciênc. Saúde Coletiva [Internet]. 2019 [acesso em 15.ago.2020]; 24(6):2167-2172. Disponível em: https://www.scielo.br/pdf/csc/v24n6/1413-8123-csc-24-06-2167.pdf

8. Oliveira EP, Douglas W. Direito à saúde x pandemia - A judicialização em tempos de coronavírus: quando o direito encontra a realidade. 1르ed. Rio de Janeiro: Impetus; 2020. p.62.

9. Alves EF, Silva MHA, Oliveira FA, Silva TT. Vulnerabilidade social diante da fosfoetanolamina a partir da teoria principialista. Revista Bioética [Internet]. 2019 [acesso em 16.ago.2020]; 27(1):173-8. Disponível em: https://www.scielo.br/pdf/bioet/v27n1/19838042-bioet-27-01-0173.pdf

10. Brasil. Ministério da Saúde. Orientações do Ministério da Saúde para Manuseio Medicamentoso Precoce de Pacientes com Diagnóstico da Covid-19 [Internet]. Brasília, 20 mai 2020 [acesso em 10.jul.2020]. Disponível em: 
https://saude.gov.br/images/pdf/2020/june/17/orienta----es-d-para-manuseiomedicamentoso-precoce-de-pacientes-com-diagn--stico-da-covid-19.pdf

11. Brasil. Ministério da Saúde. Nota Técnica n 66, de 30 de março de 2020 [Internet]. Analisa a possibilidade de incluir as substâncias cloroquina e hidroxicloroquina em lista do Anexo I da Portaria SVS/MS no 344/1998. Brasília, 2020 [acesso em 4.jun.2020]. Disponível em:

http://portal.anvisa.gov.br/documents/10181/5821187/NotaT\%C3\%A9cnica+sobre+inclus\% C3\%A3o+da+cloroquina+e+hidroxicloroquina+no+anexo+da+Portaria+344/6054f686-f5e34210-b5dd-67bae4502be0

12. Brasil. Ministério da Saúde. Gabinete do Ministro. Portaria no 1.554, de 30 de julho de 2013. Dispõe sobre as regras de financiamento e execução do Componente Especializado da Assistência Farmacêutica no âmbito do Sistema Único de Saúde (SUS). Diário Oficial da União [da] República Federativa do Brasil. (31 jul 2013); Seção 1. p. 69-71.

13. Brasil. Ministério da Saúde. Secretaria de Vigilância em Saúde. Departamento de Imunização e Doenças Transmissíveis. Guia de tratamento da malária no Brasil [Internet]. Brasília, 2020 [acesso em 14.jun. 2020]. Disponível em: https://portalarquivos2.saude.gov.br/images/pdf/2020/janeiro/29/af-guia-tratamentomalaria-28jan20-isbn.pdf

14. Agência Nacional de Vigilância Sanitária - Anvisa. Nota Técnica sobre Cloroquina e Hidroxicloroquina [Internet]. Brasília, 2020 [acesso em 15.jun. 2020]. Disponível em: http://portal.anvisa.gov.br/documents/219201/4340788/Nota+Te\%25C2\%25B4cnica+sobre +Cloroquina+e+Hidroxicloroquina.pdf/659d0105-60cf-4cab-b80a-fa0e29e2e799

15. Caetano R, Silva RM, Pedro EM, Oliveira IAG, Navega Biz A, Santana P. Incorporação de novos medicamentos pela Comissão Nacional de Incorporação de Tecnologias do SUS, 2012 a junho de 2016. Ciênc. Saúde Coletiva [Internet]. 2017 [acesso em 10.out.2019]; 22(8):2513-2525. Disponível em: https://www.scielo.br/pdf/csc/v22n8/1413-8123-csc-22-082513.pdf

16. Lopes LMN, Coelho TL, Diniz SD, Andrade EIG. Integralidade e universalidade da assistência farmacêutica em tempos de judicialização da saúde. Saúde e Sociedade [Internet]. 2019 [acesso em 26.nov.2019]; 28(2):124-131. Disponível em: https://www.scielo.br/pdf/sausoc/v28n2/1984-0470-sausoc-28-02-124.pdf

17. Campolina AG, Soárez PC, Amaral FV, Abe JM. Análise de decisão multicritério para alocação de recursos e avaliação de tecnologias em saúde: tão longe e tão perto? Cd. Saúde Pública [Internet]. 2017 [acesso em 28.jan.2020]; 33(10):1-15. Disponível em: https://www.scielo.br/pdf/csp/v33n10/1678-4464-csp-33-10-e00045517.pdf

18. Menezes APR, Moretti B, Reis AAC. O futuro do SUS: impactos das reformas neoliberais na saúde pública - austeridade versus universalidade. Saúde em Debate [Internet]. 2019 [acesso em 13.jul.2020]; 43(5):58-70. Disponível em:

https://scielosp.org/pdf/sdeb/2019.v43nspe5/58-70/pt 
19. Carneiro DF. Direito à saúde e judicialização da assistência farmacêutica. Revista de Doutrina e Jurisprudência [Internet]. 2017 [acesso em 15.jul.2020]; 108(2):242-256.

Disponível em: https://revistajuridica.tjdft.jus.br/index.php/rdj/article/view/50/51

20. Maduro LCS, Pereira LRL. Processos judiciais para obter medicamentos em Ribeirão Preto. Revista Bioética [Internet]. 2020 [acesso em 10.jul.2020]; 28(1):166-72. Disponível em: https://www.scielo.br/pdf/bioet/v28n1/1983-8042-bioet-28-01-0166.pdf

21. Sousa EDG, Franciso AH, Alfredo E, Manchola C. Termos de esclarecimento e responsabilidade à luz da bioética de intervenção. Revista Bioética [Internet]. 2018 [acesso em 20.ago.2020]; 26(3):360-70. Disponível em: https://www.scielo.br/pdf/bioet/v26n3/19838042-bioet-26-03-0360.pdf

22. Silva $A C$, Rezende D. A relação entre o princípio da autonomia e o princípio da beneficência (e não-maleficência) na bioética médica. Revista Brasileira de Estudos Políticos [Internet]. 2017 [acesso em 18.jul.2020]; 115:13-45. Disponível em:

https://pos.direito.ufmg.br/rbep/index.php/rbep/article/view/514

23. Silva CF, Ventura M, Castro CGSO. Perspectivas bioéticas sobre justiça nos ensaios clínicos. Revista Bioética [Internet]. 2016 [acesso em 22.ago.2020]; 24(2):292-303. Disponível em: https://www.scielo.br/pdf/bioet/v24n2/1983-8034-bioet-24-2-0292.pdf

24. Neto OHC, Gonçalves LAO, Andrade EIG. A judicialização da Saúde na percepção de médicos prescritores [Internet]. 2018 [acesso em 03.nov.2019]; 22(64):165-76. Disponível em: https://www.scielo.br/pdf/icse/v22n64/1807-5762-icse-1807-576220160314.pdf

\section{Colaboradores}

Oliveira P, Viana CCV, Campos Neto OH, Zocratto KBF contribuíram para a concepção/desenho do artigo; análise e interpretação de dados; redação do artigo; revisão crítica e aprovação da versão final. Pereira GG e Santos AS contribuíram para a aprovação da versão final do artigo. 